\title{
Anatomical Variations of the Cephalic Vein at its termination in a Group of Sri Lankan Subjects: A Cadaveric study
}

\author{
Samarawickrama $\mathrm{MB}^{1}$ *, Rodrigo $\mathrm{PM}^{1}$ \\ ${ }^{\prime}$ Department of Anatomy, Faculty of Medicine, University Ruhuna, Sri Lanka. \\ *samaramb@gmail.com
}

The cephalic vein (CV) in the deltopectoral groove is used for central venous access with lower incidence of complications than other sites.

However, the presence of variations at this site would cause side effects like bleeding and misplacement of implant devices. We examined CVs at the deltopectoral groove in cadavers during educational dissection in the Department of Anatomy, Faculty of Medicine, University of Ruhuna. Any variation of the $\mathrm{CV}$ termination and relation to the clavicle was noted. The study included $64 \mathrm{CVs}$ in 32 cadavers. There were 18 males and 14 females. Two female cadavers had unilateral variations while one male had bilateral variations of the $\mathrm{CV}$. Two different types of variations were identified among these four CVs. The first variation was the presence of a persistent jugulocephalic vein where the $\mathrm{CV}$ drains into external jugular vein (EJV) after crossing the clavicle. This variation was seen in the left side of a female cadaver and the right side of the male cadaver. The other variation was the presence of a bifid cephalic arch where $\mathrm{CV}$ terminates in the axillary vein and an additional communication with the EJV. This was seen in right side of a female cadaver and the left side of the male cadaver. In these four instances the cephalic vein or the communicating vein crosses the clavicle lateral to the midpoint of the clavicle. Persistent jugulocephalic vein and bifid cephalic arch were the variations noted in this study. Awareness of these variations and their incidence are important for radiologists, vascular surgeons and interventional cardiologists to avoid complications during vascular interventional procedures. 